\title{
The boundedness of variation associated with the commutators of approximate identities
}

\author{
Yongming Wen ${ }^{1}$ and Xianming $\mathrm{Hou}^{2 *}$
}

\section{"Correspondence:}

houxianming37@163.com

${ }^{2}$ School of Mathematics and

Statistics, LinYi University, LinYi

276005, China

Full list of author information is

available at the end of the article

\section{Springer}

\begin{abstract}
In this paper, we establish $L^{P}$-boundedness and endpoint estimates for variation associated with the commutators of approximate identities, which are new for variation operators. As corollaries, we obtain the corresponding boundedness results for variation associated with the commutators of heat semigroups and Poisson semigroups.
\end{abstract}

MSC: 42B20; 42B25; 42B30; 42B35

Keywords: Variation; Boundedness; Commutators

\section{Introduction and main results}

The intension of this paper is to obtain boundedness of variation associated with the commutators of approximate identities. Before stating our results, we first recall some relevant background.

The boundedness of commutators of singular integrals, especially the endpoint estimates, attracts lots of scholars' interest. Recall that given a locally integral function $b$ and a linear or nonlinear operator $T$, the commutator $[b, T]$ is defined by

$$
[b, T] f(x):=T((b(x)-b(\cdot)) f)(x) .
$$

And we say that $b$ belongs to $\mathrm{BMO}\left(\mathbb{R}^{\mathrm{n}}\right)$ spaces if

$$
\|b\|_{\mathrm{BMO}\left(\mathbb{R}^{\mathrm{n}}\right)}:=\sup _{Q} \frac{1}{|Q|} \int_{Q}\left|b(x)-\langle b\rangle_{Q}\right| d x<\infty .
$$

Coifman, Rochberg, and Weiss [8] first studied the $L^{p}$-boundedness of commutators of singular integrals with the symbol $b \in \mathrm{BMO}\left(\mathbb{R}^{\mathrm{n}}\right)$. While the endpoint case $p=1$ was the remarkable work of Pérez [23], it was shown in [23] that the commutators of CalderónZygmund operators enjoy an $L \log L$ estimate instead of being of weak type $(1,1)$. Moreover, it was observed in [22] that the commutators do not always map $H^{1}\left(\mathbb{R}^{n}\right)$ into $L^{1}\left(\mathbb{R}^{n}\right)$. In fact, Pérez [23] proved that the commutators of Calderón-Zygmund operators are

(c) The Author(s) 2021. This article is licensed under a Creative Commons Attribution 4.0 International License, which permits use, sharing, adaptation, distribution and reproduction in any medium or format, as long as you give appropriate credit to the original author(s) and the source, provide a link to the Creative Commons licence, and indicate if changes were made. The images or other third party material in this article are included in the article's Creative Commons licence, unless indicated otherwise in a credit line to the material. If material is not included in the article's Creative Commons licence and your intended use is not permitted by statutory regulation or exceeds the permitted use, you will need to obtain permission directly from the copyright holder. To view a copy of this licence, visit http://creativecommons.org/licenses/by/4.0/. 
bounded from $\mathcal{H}_{b}^{1}\left(\mathbb{R}^{n}\right)$ (see its definition in [23]) into $L^{1}\left(\mathbb{R}^{n}\right)$. Precisely, Pérez proved this result by establishing that

$$
\sup \left\{\|[b, T] a\|_{L^{1}\left(\mathbb{R}^{n}\right)}\right\}<\infty,
$$

where $a$ is a $b$-atom (see its definition in [23]). However, Ky [15] pointed out that the above inequality does not suffice to illustrate that $[b, T]$ is bounded from $\mathcal{H}_{b}^{1}\left(\mathbb{R}^{n}\right)$ into $L^{1}\left(\mathbb{R}^{n}\right)$. Ky filled this gap and proved that $[b, T]$ is bounded from $H_{b}^{1}\left(\mathbb{R}^{n}\right)$ into $L^{1}\left(\mathbb{R}^{n}\right)$, where $H_{b}^{1}\left(\mathbb{R}^{n}\right)$ (see Definition 2.4) contains $\mathcal{H}_{b}^{1}\left(\mathbb{R}^{n}\right)$.

In this paper, we study variation associated with the commutators of approximate identities. We first recall some definitions, notations, and background. Let $I \subset \mathbb{R}^{+}$and $\mathfrak{a}:=\left\{a_{t}\right\}_{t \in I}$ be a family of complex numbers. For $\rho>2$, the $\rho$-variation of $\mathfrak{a}$ is defined by

$$
\|\mathfrak{a}\|_{\mathcal{V}_{\rho}}:=\sup \left(\sum_{k \geq 1}\left|a_{t_{k}}-a_{t_{k+1}}\right|^{\rho}\right)^{1 / \rho}
$$

where the supremum is taken over all finite decreasing sequences $\left\{t_{k}\right\}$ in $I$. Let $\mathcal{F}:=\left\{F_{t}\right\}_{t>0}$ be a family of operators. We also define the $\rho$-variation of the family $\mathcal{F}$ by

$$
\mathcal{V}_{\rho}(\mathcal{F} f)(x):=\left\|\left\{F_{t} f(x)\right\}_{t>0}\right\|_{\mathcal{V}_{\rho}}
$$

Let $\phi \in \mathcal{S}\left(\mathbb{R}^{n}\right)$ satisfy $\int_{\mathbb{R}^{n}} \phi(x) d x=1$, where $\mathcal{S}\left(\mathbb{R}^{n}\right)$ is the space of Schwartz functions. We consider the following family of operators:

$$
\Phi \star f(x):=\left\{\phi_{t} * f(x)\right\}_{t>0},
$$

where $\phi_{t}(x):=t^{-n} \phi(x / t)$. Let $b \in L_{\mathrm{loc}}^{1}\left(\mathbb{R}^{n}\right)$. We will also take into account the corresponding family of commutators of operators

$$
(\Phi \star f)_{b}(x):=\left\{b(x)\left(\phi_{t} * f\right)(x)-\phi_{t} *(b f)(x)\right\}_{t>0},
$$

where

$$
b(x)\left(\phi_{t} * f\right)(x)-\phi_{t} *(b f)(x)=\int_{\mathbb{R}^{n}} \frac{1}{t^{n}} \phi\left(\frac{x-y}{t}\right)(b(x)-b(y)) f(y) d y .
$$

The $\rho$-variation of families $\Phi \star f$ and $(\Phi \star f)_{b}$ is defined respectively by

$$
\mathcal{V}_{\rho}(\Phi \star f)(x)=\sup _{\left\{t_{k}\right\} \downarrow 0}\left(\sum_{k \geq 1}\left|\phi_{t_{k}} * f(x)-\phi_{t_{k+1}} * f(x)\right|^{\rho}\right)^{1 / \rho}
$$

and

$$
\begin{aligned}
\mathcal{V}_{\rho}\left((\Phi \star f)_{b}\right)(x)= & \sup _{\left\{t_{k}\right\} \downarrow 0}\left(\sum_{k \geq 1} \mid b(x)\left(\phi_{t_{k}} * f\right)(x)-\phi_{t_{k}} *(b f)(x)\right. \\
& \left.-b(x)\left(\phi_{t_{k+1}} * f\right)(x)+\left.\phi_{t_{k+1}} *(b f)(x)\right|^{\rho}\right)^{1 / \rho} .
\end{aligned}
$$


In [16], Lépingle improved the well-known Doob maximal inequality by proving a variational inequality for martingales. Relying upon this result, Bourgain [2] obtained corresponding variational estimates for the Birkhoff ergodic averages and pointwise convergence results. This work has set up a new research subject in harmonic analysis and ergodic theory. Afterwards, the study of variational inequalities has been spilled over into harmonic analysis, probability and ergodic theory. Particularly, we refer readers to see [5] for the classical work of $\rho$-variation operators for singular integrals. In [5], the authors gave the $L^{p}$-bounds and weak type $(1,1)$ bounds for $\rho$-variation operators of truncated Hilbert transform if $\rho>2$, and then they extended the results to higher dimension in [6]. For results of rough kernels and weighted cases, see [10, 13, 20]. In 2009, Crescimbeni et al. [9] established the $L^{p}$-bounds and weak type $(1,1)$ bounds for variation operators of heat semigroups and Poisson semigroups. Betancor et al. [1] also considered the $L^{p}$ boundedness of variation operators of heat semigroups associated with Schrödinger operators, which was generalized to metric spaces endowed with nonnegative Borel measure by Bui [4] and to weighted cases by Tang and Zhang [25]. The acting on Hardy spaces for variation operators associated with approximate identities, which include the variation operators of heat semigroups, was given by Liu [19]. For recent works on variation inequalities, we refer readers to [3, 11, 21, 26, 27].

The other side of the shield, the variational inequalities for the commutators of singular integrals also have been intensively studied. In 2013, Betancor et al. [1] studied the mapping property of variation operators for the commutators of Riesz transforms in the Euclidean and Schrödinger setting. A few years later, Liu and $\mathrm{Wu}$ [18] obtained the weighted $L^{p}$-boundedness for variation operators of commutators of truncated singular integrals with the Calderón-Zygmund kernels. Recently, variation operators of commutators with rough kernels were also obtained in [7]. For more works on variation operators of commutators, see [17, 28, 29]

From the known results about variation inequalities, none of the boundedness estimates for variation of commutators have been established, can we establish the corresponding boundedness results for variation of commutators? In this paper, we give a firm answer to this question.

Our contribution of this paper is the following.

Theorem 1.1 Let $\phi \in \mathcal{S}\left(\mathbb{R}^{n}\right)$ satisfy $\int_{\mathbb{R}^{n}} \phi(x) d x=1, b \in \mathrm{BMO}\left(\mathbb{R}^{\mathrm{n}}\right)$, and $1<p<\infty$, then for $\rho>2$ and $f \in L^{p}\left(\mathbb{R}^{n}\right), \mathcal{V}_{\rho}\left((\Phi \star f)_{b}\right)$ is bounded on $L^{p}\left(\mathbb{R}^{n}\right)$.

Theorem 1.2 Let $\phi \in \mathcal{S}\left(\mathbb{R}^{n}\right)$ satisfy $\int_{\mathbb{R}^{n}} \phi(x) d x=1$ and $b \in \operatorname{BMO}\left(\mathbb{R}^{\mathrm{n}}\right)$, then for $\rho>2$ and $f \in H^{1}\left(\mathbb{R}^{n}\right), \mathcal{V}_{\rho}\left((\Phi \star f)_{b}\right)$ is bounded from $H^{1}\left(\mathbb{R}^{n}\right)$ to $L^{1, \infty}\left(\mathbb{R}^{n}\right)$.

Theorem 1.3 Let $\phi \in \mathcal{S}\left(\mathbb{R}^{n}\right)$ satisfy $\int_{\mathbb{R}^{n}} \phi(x) d x=1$ and $b \in \mathrm{BMO}\left(\mathbb{R}^{\mathrm{n}}\right)$, then for $\rho>2$ and $f \in H_{b}^{1}\left(\mathbb{R}^{n}\right), \mathcal{V}_{\rho}\left((\Phi \star f)_{b}\right)$ is bounded from $H_{b}^{1}\left(\mathbb{R}^{n}\right)$ to $L^{1}\left(\mathbb{R}^{n}\right)$.

Specially, we will consider the families of operators

$$
\mathcal{H}:=\left\{e^{t \Delta} f(x)\right\}_{t>0}, \quad \mathcal{P}:=\left\{e^{-t \sqrt{-\Delta}} f(x)\right\}_{t>0}
$$


where $\Delta=\sum_{i=1}^{n} \frac{\partial^{2}}{\partial x_{i}^{2}}$, the heat semigroup $e^{t \Delta}$ and the Poisson semigroup $e^{-t \sqrt{-\Delta}}$ are defined as

$$
\begin{aligned}
& e^{t \Delta} f(x)=(\pi t)^{-n / 2} \int_{\mathbb{R}^{n}} e^{-|x-y|^{2} / t} f(y) d y, \\
& e^{-t \sqrt{-\Delta}} f(x)=\frac{1}{\sqrt{4 \pi}} \int_{0}^{\infty} t e^{-t^{2} /(4 s)} e^{s \Delta} f(x) s^{-3 / 2} d s=\frac{1}{\sqrt{\pi}} \int_{0}^{\infty} \frac{e^{-u}}{\sqrt{u}} e^{t^{2} /(4 u) \Delta} f(x) d u .
\end{aligned}
$$

And we will also take into account the corresponding families of commutators of operators

$$
\mathcal{H}_{b}:=\left\{e^{t \Delta}((b(x)-b(\cdot)) f)(x)\right\}_{t>0}, \quad \mathcal{P}_{b}:=\left\{e^{-t \sqrt{-\Delta}}((b(x)-b(\cdot)) f)(x)\right\}_{t>0},
$$

where $e^{t \Delta}((b(x)-b(\cdot)) f)$ and $e^{-t \sqrt{-\Delta}}((b(x)-b(\cdot)) f)$ are defined by

$$
\begin{aligned}
& e^{t \Delta}((b(x)-b(\cdot)) f)(x)=(\pi t)^{-n / 2} \int_{\mathbb{R}^{n}} e^{-|x-y|^{2} / t}(b(x)-b(y)) f(y) d y, \\
& e^{-t \sqrt{-\Delta}}((b(x)-b(\cdot)) f)(x)=\pi^{-1 / 2} \int_{0}^{\infty} e^{-u} u^{-1 / 2} e^{t^{2} /(4 u) \Delta}((b(x)-b(\cdot)) f)(x) d u .
\end{aligned}
$$

Similar to (1.3) and (1.4), we can define the $\rho$-variation of families $\mathcal{H}, \mathcal{H}_{b}, \mathcal{P}$, and $\mathcal{P}_{b}$, and we denote them by $\mathcal{V}_{\rho}(\mathcal{H}), \mathcal{V}_{\rho}\left(\mathcal{H}_{b}\right), \mathcal{V}_{\rho}(\mathcal{P})$, and $\mathcal{V}_{\rho}\left(\mathcal{P}_{b}\right)$, respectively. It is well known that the kernel of the heat semigroup

$$
\phi_{t}(x)=(\pi t)^{-n / 2} e^{-|x|^{2} / t}
$$

belongs to $\mathcal{S}\left(\mathbb{R}^{n}\right)$ and satisfies $\int_{\mathbb{R}^{n}} \phi(x) d x=1$. Following the steps in proving Theorem 1.2 and Theorem 1.3, the endpoint estimates also hold for variation associated with the commutators of Poisson semigroups. Therefore, we have the following corollary.

Corollary 1.4 Let $b \in \mathrm{BMO}\left(\mathbb{R}^{\mathrm{n}}\right)$, then for $\rho>2$,

(1) $\mathcal{V}_{\rho}\left(\mathcal{H}_{b}\right)$ is bounded on $L^{p}\left(\mathbb{R}^{n}\right)$;

(2) $\mathcal{V}_{\rho}\left(\mathcal{H}_{b}\right)$ is bounded from $H^{1}\left(\mathbb{R}^{n}\right)$ to $L^{1, \infty}\left(\mathbb{R}^{n}\right)$;

(3) $\mathcal{V}_{\rho}\left(\mathcal{H}_{b}\right)$ is bounded from $H_{b}^{1}\left(\mathbb{R}^{n}\right)$ to $L^{1}\left(\mathbb{R}^{n}\right)$.

The same conclusions also hold for $\mathcal{V}_{\rho}\left(\mathcal{P}_{b}\right)$.

Remark 1.5 Given a family of operators $\mathcal{F}=\left\{F_{\epsilon}\right\}_{\epsilon>0}$, define the oscillation operator by

$$
\mathcal{O}(\mathcal{F} f)(x)=\left(\sum_{i=1}^{\infty} \sup _{t_{i+1} \leq \epsilon_{i}<\epsilon_{i+1} \leq t_{i}}\left|F_{\epsilon_{i}} f(x)-F_{\epsilon_{i+1}} f(x)\right|^{2}\right)^{1 / 2}
$$

with $\left\{t_{i}\right\}$ being a fixed sequence decreasing to 0 . We remark that from the proofs of the above theorems, the corresponding results also hold for the oscillation associated with the commutators of approximate identities.

Remark 1.6 For a family of operators given in Remark 1.5, define the $\alpha$-jump operator for $\mathcal{F}$ at $x$ by

$$
N_{\alpha}(\mathcal{F} f)(x):=\sup \left\{N \in \mathbb{Z}^{+}: \exists s_{1}<\epsilon_{1} \leq s_{2}<\epsilon_{2} \leq \cdots \leq s_{N}<\epsilon_{N}\right.
$$




$$
\text { such that } \left.\left|F_{\epsilon_{k}} f(x)-F_{s_{k}} f(x)\right|>\alpha\right\} \text {. }
$$

We refer readers to $[10,14]$ for more details about jump operators. It is trivial that $\alpha\left(N_{\alpha}\left((\Phi \star f)_{b}\right)(x)\right)^{1 / \rho} \leq C_{\rho} \mathcal{V}_{\rho}\left((\Phi \star f)_{b}\right)(x)$, then the results of Theorems 1.1-1.3 also hold for $\alpha\left(N_{\alpha}\left((\Phi \star f)_{b}\right)(x)\right)^{1 / \rho}$.

We organize the rest of the paper as follows. In Sect. 2, we give some preliminaries. Section 3 is devoted to proving Theorems 1.1-1.3.

We end this section by making some conventions, we denote a positive constant by $C$, which may change at each occurrence. We also denote $f \lesssim g, f \sim g$ if $f \leq C g$ and $f \lesssim g \lesssim f$, respectively. For any ball $B:=B\left(x_{0}, r\right) \subset \mathbb{R}^{n}, x_{0}$ and $r$ are the center and the radius of $B$, respectively, and $\langle f\rangle_{B}$ means the mean value of $f$ over $B, \chi_{B}$ represents the characteristic function of $B$.

\section{Preliminaries}

In this section, we introduce some necessary definitions and lemmas.

For $\delta>0$, we denote $M_{\delta}(f):=M\left(|f|^{\delta}\right)^{1 / \delta}$, where $M$ is the Hardy-Littlewood maximal operator. The sharp maximal function $M^{\sharp}$ is defined by

$$
M^{\sharp}(f)(x)=\operatorname{supinf}_{Q \ni x} \frac{1}{|Q|} \int_{Q}|f(y)-c| d y \sim \sup _{Q \ni x} \frac{1}{|Q|} \int_{Q}\left|f(y)-\langle f\rangle_{Q}\right| d y .
$$

In this paper, we use the following famous Fefferman-Stein inequality, see [12]:

$$
\int_{\mathbb{R}^{n}} M(f)(x)^{p} d x \leq C \int_{\mathbb{R}^{n}} M^{\sharp}(f)(x)^{p} d x
$$

for all function $f$ such that the left-hand side is finite, where $0<p<\infty$.

Next, let us recall some known facts concerned with $H^{1}\left(\mathbb{R}^{n}\right)$.

\section{Definition 2.1 Denote}

$$
\mathcal{B}:=\left\{\phi \in \mathcal{S}\left(\mathbb{R}^{n}\right):|\phi(x)|+|\nabla \phi(x)| \leq\left(1+|x|^{2}\right)^{-(n+1)}\right\},
$$

where $\nabla=\left(\partial / \partial x_{1}, \ldots, \partial / \partial x_{n}\right)$. Define

$$
\mathfrak{M} f(x):=\sup _{\phi \in \mathcal{B}} \sup _{|y-x| \leq t}\left|f * \phi_{t}(y)\right|
$$

where $\phi_{t}(x)=t^{-n} \phi(x / t)$ with $t>0$. Then the space $H^{1}\left(\mathbb{R}^{n}\right)$ is the space of all tempered distributions $f$ such that $\mathfrak{M} f \in L^{1}\left(\mathbb{R}^{n}\right)$ equipped with the norm $\|f\|_{H^{1}\left(\mathbb{R}^{n}\right)}=\|\mathfrak{M} f\|_{L^{1}\left(\mathbb{R}^{n}\right)}$.

Definition 2.2 Let $B$ be a ball, we say that a function $a(x)$ is an $(1, \infty)$-atom if it satisfies:

(1) $\operatorname{supp} a \subset B$;

(2) $\|a\|_{L^{\infty}} \leq|B|^{-1}$;

(3) $\int_{B} a(x) d x=0$. 
Lemma 2.3 (Cf. [24]) A function $f \in L^{1}\left(\mathbb{R}^{n}\right)$ belongs to $H^{1}\left(\mathbb{R}^{n}\right)$ if and only iff $=\sum_{i} \lambda_{i} a_{i}$ in $H^{1}$ norm or $L^{1}$ norm, where $a_{i}$ s are $(1, \infty)$-atoms, $\lambda_{i} \in \mathbb{C}$ with $\sum_{i}\left|\lambda_{i}\right|<\infty$. Furthermore,

$$
\|f\|_{H^{1}\left(\mathbb{R}^{n}\right)} \sim \inf \left\{\sum_{i}\left|\lambda_{i}\right|\right\}
$$

where the infimum is taken over all the above atomic decomposition off.

As we stated in the introduction, the space $H_{b}^{1}\left(\mathbb{R}^{n}\right)$ is introduced to fill the gap left by Pérez [23]. We present its definition as follows.

Definition 2.4 Let $b \in \mathrm{BMO}\left(\mathbb{R}^{\mathrm{n}}\right)$. The space $H_{b}^{1}\left(\mathbb{R}^{n}\right)$ consists of all $f$ in $H^{1}\left(\mathbb{R}^{n}\right)$ such that $[b, \mathfrak{M}] f(x):=\mathfrak{M}(b(x) f(\cdot)-b(\cdot) f(\cdot))(x)$ belongs to $L^{1}\left(\mathbb{R}^{n}\right)$. The norm of $H_{b}^{1}\left(\mathbb{R}^{n}\right)$ is defined by $\|f\|_{H_{b}^{1}\left(\mathbb{R}^{n)}\right.}:=\|f\|_{H^{1}\left(\mathbb{R}^{n}\right)}\|b\|_{\mathrm{BMO}}+\|[b, \mathfrak{M}] f\|_{L^{1}\left(\mathbb{R}^{n}\right)}$.

In [15], Ky gave the following criterion to verify the mapping property of operators on Hardy type spaces.

Lemma 2.5 (Cf. [15]) Let $b \in \mathrm{BMO}\left(\mathbb{R}^{\mathrm{n}}\right)$, $T$ be a linear or nonlinear operator, and the commutator be defined as (1.1). If $T$ is bounded from $H^{1}\left(\mathbb{R}^{n}\right)$ to $L^{1}\left(\mathbb{R}^{n}\right)$ and satisfies

$$
\left\|\left(b-\langle b\rangle_{Q}\right) \operatorname{Ta}\right\|_{L^{1}\left(\mathbb{R}^{n)}\right.} \lesssim\|b\|_{\mathrm{BMO}\left(\mathbb{R}^{n}\right)}
$$

for any $\psi$-atom a of $H^{1}\left(\mathbb{R}^{n}\right)$ with supp $a \subset Q$, then the commutator $[b, T]$ maps $H_{b}^{1}\left(\mathbb{R}^{n}\right)$ into $L^{1}\left(\mathbb{R}^{n}\right)$.

Remark 2.6 We remark that we do not give the definition of $\psi$-atom of $H^{1}\left(\mathbb{R}^{n}\right)$ since we need to state so many backgrounds of it, but only use the properties $\|a\|_{L^{2}(Q)} \leq|Q|^{-1 / 2}$ and $\int_{Q} a(y) d y=0$, where $Q$ is a cube. We refer readers to [15] for its definition.

In this paper, we also need the following lemma established in [19].

Lemma 2.7 (Cf. [19]) Let $\phi \in \mathcal{S}\left(\mathbb{R}^{n}\right)$ satisfy $\int_{\mathbb{R}^{n}} \phi(x) d x=1$ and $1<p<\infty$, then for $\rho>2$, we have

(1) $\mathcal{V}_{\rho}(\Phi \star f)$ is bounded on $L^{p}\left(\mathbb{R}^{n}\right)$;

(2) $\mathcal{V}_{\rho}(\Phi \star f)$ is bounded from $L^{1}\left(\mathbb{R}^{n}\right)$ to $L^{1, \infty}\left(\mathbb{R}^{n}\right)$;

(3) $\mathcal{V}_{\rho}(\Phi \star f)$ is bounded from $H^{1}\left(\mathbb{R}^{n}\right)$ to $L^{1}\left(\mathbb{R}^{n}\right)$.

\section{The boundedness of commutators}

To prove Theorem 1.1, we first establish the following lemma.

Lemma 3.1 Let $\phi \in \mathcal{S}\left(\mathbb{R}^{n}\right)$ satisfy $\int_{\mathbb{R}^{n}} \phi(x) d x=1$ and $b \in \mathrm{BMO}\left(\mathbb{R}^{\mathrm{n}}\right)$, then for $\rho>2$ and any $1<s<\infty$, there is a constant $C>0$ such that

$$
M^{\sharp}\left(\mathcal{V}_{\rho}\left((\Phi \star f)_{b}\right)\right)(x) \leq C\|b\|_{\mathrm{BMO}\left(\mathbb{R}^{\mathrm{n}}\right)}\left[M_{s}\left(\mathcal{V}_{\rho}(\Phi \star f)\right)(x)+M_{s}(f)(x)\right] .
$$


Proof For $x \in \mathbb{R}^{n}$, let $B:=B\left(x_{0}, r\right)$ be any ball containing $x$. We write $f=f_{1}+f_{2}$, where $f_{1}=f \chi_{4 B}$. For $y \in B$, one can see that

$$
\begin{aligned}
\mathcal{V}_{\rho}\left((\Phi \star f)_{b}\right)(y) \leq & \left|b(y)-\langle b\rangle_{4 B}\right| \mathcal{V}_{\rho}(\Phi \star f)(y)+\mathcal{V}_{\rho}\left(\Phi \star\left(\left(b-\langle b\rangle_{4 B}\right) f_{1}\right)\right)(y) \\
& +\mathcal{V}_{\rho}\left(\Phi \star\left(\left(b-\langle b\rangle_{4 B}\right) f_{2}\right)\right)(y) .
\end{aligned}
$$

Then

$$
\begin{aligned}
& \frac{1}{|B|} \int_{B}\left|\mathcal{V}_{\rho}\left((\Phi \star f)_{b}\right)(y)-\mathcal{V}_{\rho}\left(\Phi \star\left(\left(b-\langle b\rangle_{4 B}\right) f_{2}\right)\right)\left(x_{0}\right)\right| d y \\
& \leq \frac{1}{|B|} \int_{B}\left|b(y)-\langle b\rangle_{4 B}\right| \mathcal{V}_{\rho}(\Phi \star f)(y) d y \\
&+\frac{1}{|B|} \int_{B} \mathcal{V}_{\rho}\left(\Phi \star\left(\left(b-\langle b\rangle_{4 B}\right) f_{1}\right)\right)(y) d y \\
&+\frac{1}{|B|} \int_{B}\left|\mathcal{V}_{\rho}\left(\Phi \star\left(\left(b-\langle b\rangle_{4 B}\right) f_{2}\right)\right)(y)-\mathcal{V}_{\rho}\left(\Phi \star\left(\left(b-\langle b\rangle_{4 B}\right) f_{2}\right)\right)\left(x_{0}\right)\right| d y \\
&= I+I I+I I I .
\end{aligned}
$$

We first estimate $I$. Using Hölder's inequality with exponents $s$ and $s^{\prime}$, then

$$
\begin{aligned}
I & \leq\left(\frac{1}{|B|} \int_{B}\left|b(y)-\langle b\rangle_{4 B}\right|^{s^{\prime}} d y\right)^{1 / s^{\prime}}\left(\frac{1}{|B|} \int_{B} \mathcal{V}_{\rho}(\Phi \star f)(y)^{s} d y\right)^{1 / s} \\
& \lesssim\|b\|_{\mathrm{BMO}\left(\mathbb{R}^{\mathrm{n}}\right)} M_{s}\left(\mathcal{V}_{\rho}(\Phi \star f)\right)(x) .
\end{aligned}
$$

To deal with $I I$, choose $1<\mu, q<\infty$ such that $\mu q=s$. Thus, Lemma 2.7 yields that

$$
\begin{aligned}
I I & \lesssim\left(\frac{1}{|B|} \int_{B} \mathcal{V}_{\rho}\left(\Phi \star\left(\left(b-\langle b\rangle_{4 B}\right) f_{1}\right)\right)(y)^{q} d y\right)^{1 / q} \\
& \lesssim\left(\frac{1}{|B|} \int_{4 B}\left|b(y)-\langle b\rangle_{4 B}\right|^{q}|f(y)|^{q} d y\right)^{1 / q} \\
& \leq\left(\frac{1}{|B|} \int_{4 B}\left|b(y)-\langle b\rangle_{4 B}\right|^{q \mu^{\prime}} d y\right)^{1 /\left(q \mu^{\prime}\right)}\left(\frac{1}{|B|} \int_{4 B}|f(y)|^{q \mu} d y\right)^{1 /(q \mu)} \\
& \lesssim\|b\|_{\mathrm{BMO}\left(\mathbb{R}^{\mathrm{n}}\right)} M_{S} f(x) .
\end{aligned}
$$

Finally, we consider III. Note that

$$
\begin{aligned}
&\left|\mathcal{V}_{\rho}\left(\Phi \star\left(\left(b-\langle b\rangle_{4 B}\right) f_{2}\right)\right)(y)-\mathcal{V}_{\rho}\left(\Phi \star\left(\left(b-\langle b\rangle_{4 B}\right) f_{2}\right)\right)\left(x_{0}\right)\right| \\
& \leq \leq\left\|\left\{\phi_{t} *\left(\left(b-\langle b\rangle_{4 B}\right) f_{2}\right)(y)-\phi_{t} *\left(\left(b-\langle b\rangle_{4 B}\right) f_{2}\right)\left(x_{0}\right)\right\}_{t>0}\right\|_{\mathcal{V}_{\rho}} \\
&= \sup _{t_{k} \downarrow 0}\left(\sum_{k} \mid \int_{\mathbb{R}^{n} \backslash 4 B}\left\{\left[\phi_{t_{k}}(y-z)-\phi_{t_{k+1}}(y-z)\right]-\left[\phi_{t_{k}}\left(x_{0}-z\right)-\phi_{t_{k+1}}\left(x_{0}-z\right)\right]\right\}\right. \\
& \quad\left.\times\left.\left(b(z)-\langle b\rangle_{4 B}\right) f(z) d z\right|^{\rho}\right)^{1 / \rho} \\
& \leq \int_{\mathbb{R}^{n} \backslash 4 B}|f(z)|\left|b(z)-\langle b\rangle_{4 B}\right|\left\|\left\{\phi_{t}(y-z)-\phi_{t}\left(x_{0}-z\right)\right\}_{t>0}\right\|_{\mathcal{V}_{\rho}} d z .
\end{aligned}
$$


For $z \in \mathbb{R}^{n} \backslash 4 B$, applying the mean value theorem, we get that

$$
\begin{aligned}
& \left\|\left\{\phi_{t}(y-z)-\phi_{t}\left(x_{0}-z\right)\right\}_{t>0}\right\|_{\mathcal{V}_{\rho}} \\
& \quad \leq \sup _{t_{k} \downarrow 0}\left(\sum_{k}\left|\int_{t_{k+1}}^{t_{k}} \frac{\partial}{\partial t}\left(\phi_{t}(y-z)-\phi_{t}\left(x_{0}-z\right)\right) d t\right|\right) \\
& \quad \leq \int_{0}^{\infty}\left|\frac{\partial}{\partial t}\left(\phi_{t}(y-z)-\phi_{t}\left(x_{0}-z\right)\right)\right| d t \\
& \quad \lesssim\left|y-x_{0}\right| \int_{0}^{\infty} \frac{1}{t^{n+2}}\left(1+\frac{\left|z-x_{0}\right|}{t}\right)^{-(n+2)} d t \\
& \quad=\frac{\left|y-x_{0}\right|}{\left|z-x_{0}\right|^{n+1}} \int_{0}^{\infty} \frac{t^{n}}{(t+1)^{n+2}} d t \sim \frac{\left|y-x_{0}\right|}{\left|z-x_{0}\right|^{n+1}} .
\end{aligned}
$$

Thus,

$$
\begin{aligned}
& \left|\mathcal{V}_{\rho}\left(\Phi \star\left(\left(b-\langle b\rangle_{4 B}\right) f_{2}\right)\right)(y)-\mathcal{V}_{\rho}\left(\Phi \star\left(\left(b-\langle b\rangle_{4 B}\right) f_{2}\right)\right)\left(x_{0}\right)\right| \\
& \quad \lesssim \int_{\mathbb{R}^{n} \backslash 4 B}|f(z)|\left|b(z)-\langle b\rangle_{4 B}\right| \frac{\left|y-x_{0}\right|}{\left|z-x_{0}\right|^{n+1}} d z .
\end{aligned}
$$

Observe that

$$
\begin{aligned}
& \left(\int_{\mathbb{R}^{n} \backslash 4 B} \frac{|f(z)|^{s}}{\left|z-x_{0}\right|^{n+1}} d z\right)^{1 / s} \\
& \quad \leq\left(\sum_{j=2}^{\infty} \int_{2^{j+1} B \backslash 2^{j} B} \frac{|f(z)|^{s}}{\left|z-x_{0}\right|^{n+1}} d z\right)^{1 / s} \\
& \quad \leq\left(\sum_{j=2}^{\infty}\left(2^{j} r\right)^{-n-1} \int_{2^{j+1} B}|f(z)|^{s} d z\right)^{1 / s} \lesssim r^{-1 / s} M_{s}(f)(x) .
\end{aligned}
$$

And note that

$$
\begin{aligned}
& \left(\int_{\mathbb{R}^{n} \backslash 4 B} \frac{\left|b(z)-\langle b\rangle_{B}\right|^{s^{\prime}}}{\left|z-x_{0}\right|^{n+1}} d z\right)^{1 / s^{\prime}} \\
& \quad=\left(\sum_{j=2}^{\infty} \int_{2^{j+1} B \backslash 2^{j} B} \frac{\left|b(z)-\langle b\rangle_{B}\right|^{s^{\prime}}}{\left|z-x_{0}\right|^{n+1}} d z\right)^{1 / s^{\prime}} \\
& \quad \lesssim\left(\sum_{j=2}^{\infty} \frac{1}{\left(2^{j+1} r\right)^{n+1}} \int_{2^{j+1} B \backslash 2^{j} B}\left|b(z)-\langle b\rangle_{B}\right|^{s^{\prime}} d z\right)^{1 / s^{\prime}} \\
& \quad \lesssim r^{-1 / s^{\prime}}\left[\sum_{j=2}^{\infty} 2^{-j-1}\left(\frac{1}{\left|2^{j+1} B\right|} \int_{2^{j+1} B}\left|b(z)-\langle b\rangle_{2^{j+1} B}\right|^{s^{\prime}} d z+\left|\langle b\rangle_{2^{j+1} B}-\langle b\rangle_{B}\right|^{s^{\prime}}\right)\right]^{1 / s^{\prime}} \\
& \quad \lesssim r^{-1 / s^{\prime}}\|b\|_{\mathrm{BMO}\left(\mathbb{R}^{\mathrm{n}}\right)}\left(\sum_{j=2}^{\infty} 2^{-j-1}(1+(j+1))\right) \sim r^{-1 / s^{\prime}}\|b\|_{\mathrm{BMO}\left(\mathbb{R}^{\mathrm{n}}\right) .}
\end{aligned}
$$


Combining with the above two estimates, we conclude that

$$
\begin{aligned}
& \left|\mathcal{V}_{\rho}\left(\Phi \star\left(\left(b-\langle b\rangle_{4 B}\right) f_{2}\right)\right)(y)-\mathcal{V}_{\rho}\left(\Phi \star\left(\left(b-\langle b\rangle_{4 B}\right) f_{2}\right)\right)\left(x_{0}\right)\right| \\
& \quad \leq r\left(\int_{\mathbb{R}^{n} \backslash 4 B} \frac{|f(z)|^{s}}{\left|z-x_{0}\right|^{n+1}} d z\right)^{1 / s}\left(\int_{\mathbb{R}^{n} \backslash 4 B} \frac{\left|b(z)-\langle b\rangle_{4 B}\right|^{s^{\prime}}}{\left|z-x_{0}\right|^{n+1}} d z\right)^{1 / s^{\prime}} \\
& \quad \lesssim r r^{-1 / s^{\prime}} r^{-1 / s}\|b\|_{\mathrm{BMO}\left(\mathbb{R}^{\mathrm{n}}\right)} M_{s}(f)(x)=\|b\|_{\mathrm{BMO}\left(\mathbb{R}^{\mathrm{n}}\right)} M_{s}(f)(x),
\end{aligned}
$$

which implies that

$$
I I I \lesssim\|b\|_{\mathrm{BMO}\left(\mathbb{R}^{\mathrm{n}}\right)} M_{s}(f)(x) .
$$

This together with the estimate of $I$ and $I I$ completes our proof.

Now, we are in the position to prove Theorem 1.1.

Proof of Theorem 1.1 Assume that $\left\|M\left(\mathcal{V}_{\rho}\left((\Phi \star f)_{b}\right)\right)\right\|_{L^{p}\left(\mathbb{R}^{n}\right)}$ is finite, then for $f \in C_{c}^{\infty}\left(\mathbb{R}^{n}\right)$ and any $s$ satisfying $1<s<p$, we make use of the Fefferman-Stein inequality, Lemma 3.1, and Lemma 2.7 to get that

$$
\begin{aligned}
\left\|\mathcal{V}_{\rho}\left((\Phi \star f)_{b}\right)\right\|_{L^{p}\left(\mathbb{R}^{n}\right)} & \lesssim\left\|M\left(\mathcal{V}_{\rho}\left((\Phi \star f)_{b}\right)\right)\right\|_{L^{p}\left(\mathbb{R}^{n}\right)} \\
& \lesssim\left\|M^{\sharp}\left(\mathcal{V}_{\rho}\left((\Phi \star f)_{b}\right)\right)\right\|_{L^{p}\left(\mathbb{R}^{n}\right)} \\
& \lesssim\|b\|_{\mathrm{BMO}\left(\mathbb{R}^{\mathrm{n}}\right)}\left\|M_{s}\left(\mathcal{V}_{\rho}(\Phi \star f)\right)\right\|_{L^{p}\left(\mathbb{R}^{n}\right)}+\|b\|_{\mathrm{BMO}\left(\mathbb{R}^{\mathrm{n}}\right)}\left\|M_{s}(f)\right\|_{L^{p}\left(\mathbb{R}^{n}\right)} \\
& \lesssim\|b\|_{\mathrm{BMO}\left(\mathbb{R}^{\mathrm{n}}\right)}\|f\|_{L^{p}\left(\mathbb{R}^{n}\right)},
\end{aligned}
$$

then by density we get our result.

Now, we return to proving that $\left\|M\left(\mathcal{V}_{\rho}\left((\Phi \star f)_{b}\right)\right)\right\|_{L^{p}\left(\mathbb{R}^{n}\right)}$ is finite, where $f \in C_{c}^{\infty}\left(\mathbb{R}^{n}\right)$. By the $L^{p}$-boundedness of $M$, we only need to verify that $\left\|\mathcal{V}_{\rho}\left((\Phi \star f)_{b}\right)\right\|_{L^{p}\left(\mathbb{R}^{n}\right)}$ is finite, where $f \in C_{c}^{\infty}\left(\mathbb{R}^{n}\right)$. If $b$ is a bounded function, then by Lemma 2.7 , we have

$$
\begin{aligned}
\left\|\mathcal{V}_{\rho}\left((\Phi \star f)_{b}\right)\right\|_{L^{p}\left(\mathbb{R}^{n}\right)} & \leq\|b\|_{L^{\infty}\left(\mathbb{R}^{n}\right)}\left\|\mathcal{V}_{\rho}(\Phi \star f)\right\|_{L^{p}\left(\mathbb{R}^{n}\right)}+\left\|\mathcal{V}_{\rho}(\Phi \star(b f))\right\|_{L^{p}\left(\mathbb{R}^{n}\right)} \\
& \lesssim\|b\|_{L^{\infty}\left(\mathbb{R}^{n}\right)}\|f\|_{L^{p}\left(\mathbb{R}^{n}\right)}+\|b f\|_{L^{p}\left(\mathbb{R}^{n}\right)} \\
& \lesssim\|b\|_{L^{\infty}\left(\mathbb{R}^{n}\right)}\|f\|_{L^{p}\left(\mathbb{R}^{n}\right)}<\infty
\end{aligned}
$$

While for the general BMO functions $b$, let $N \in \mathbb{N}$, define

$$
b_{N}(x)= \begin{cases}N, & b(x)>N \\ b(x), & |b(x)| \leq N \\ -N, & b(x)<-N .\end{cases}
$$

Then it is easy to check that $\left\|b_{N}\right\|_{\mathrm{BMO}\left(\mathbb{R}^{\mathrm{n}}\right)} \leq c\|b\|_{\mathrm{BMO}\left(\mathbb{R}^{\mathrm{n}}\right)}$, where the implicit constant is independent of $N$. Keeping in mind that $f \in C_{c}^{\infty}\left(\mathbb{R}^{n}\right)$ and $\phi \in \mathcal{S}\left(\mathbb{R}^{n}\right)$, using $\left|b_{N}\right| \leq|b|$ and $b \in L_{\text {loc }}^{1}\left(\mathbb{R}^{n}\right)$, by the Lebesgue dominated convergence theorem, we get

$$
\lim _{N \rightarrow \infty} \int_{\mathbb{R}^{n}}\left(b_{N}(x)-b_{N}(y)\right)\left(\phi_{t_{k}}(x-y)-\phi_{t_{k+1}}(x-y)\right) f(y) d y
$$




$$
=\int_{\mathbb{R}^{n}}(b(x)-b(y))\left(\phi_{t_{k}}(x-y)-\phi_{t_{k+1}}(x-y)\right) f(y) d y .
$$

From this, one can check that

$$
\mathcal{V}_{\rho}\left((\Phi \star f)_{b}\right) \leq \liminf _{N \rightarrow \infty} \mathcal{V}_{\rho}\left((\Phi \star f)_{b_{N}}\right)
$$

Letting $N \rightarrow \infty$ and applying the Fatou lemma, we deduce that $\left\|\mathcal{V}_{\rho}\left((\Phi \star f)_{b}\right)\right\|_{L^{p}\left(\mathbb{R}^{n}\right)}$ is finite for general BMO functions $b$.

Next, we give the proof of Theorem 1.2.

Proof of Theorem 1.2 Let $f \in H^{1}\left(\mathbb{R}^{n}\right)$, then by Lemma 2.3, we can write $f=\sum_{i} \lambda_{i} a_{i}$, where $a_{i}$ is an $(1, \infty)$ atom. To prove this conclusion, we only need to prove it for $f$ being a finite $\operatorname{sum} f=\sum_{j} \lambda_{j} a_{j}$ with $\sum_{j}\left|\lambda_{j}\right| \leq 2\|f\|_{H^{1}\left(\mathbb{R}^{n}\right)}$, where $a_{j}$ is a $(1, \infty)$ atom and $\operatorname{supp} a_{j} \subset B_{j}:=$ $B\left(x_{j}, r_{j}\right)$. In fact, assume that $\mathcal{V}_{\rho}\left((\Phi \star f)_{b}\right)$ is bounded from $H^{1}\left(\mathbb{R}^{n}\right)$ to $L^{1, \infty}\left(\mathbb{R}^{n}\right)$ for $\operatorname{such} f$, then for the general $f$, one can select a sequence $\left\{f_{k}\right\}_{k}$ with $f_{k}$ being a finite sum as above such that $f_{k}$ converges to $f$ in $H^{1}$ norm or almost everywhere when $k \rightarrow \infty$. Therefore, by a limit argument, Theorem 1.2 follows from the $L^{2}$-boundedness of $\mathcal{V}_{\rho}\left((\Phi \star f)_{b}\right)$.

Now, in the following, we assume that $f=\sum_{j} \lambda_{j} a_{j}$ is a finite sum satisfying $\sum_{j}\left|\lambda_{j}\right| \leq$ $2\|f\|_{H^{1}\left(\mathbb{R}^{n}\right)}$, where $a_{j}$ is a $(1, \infty)$ atom and supp $a_{j} \subset B_{j}$. Note that

$$
\begin{aligned}
\mathcal{V}_{\rho}\left((\Phi \star f)_{b}\right)(x) \leq & \sum_{j}\left|\lambda_{j}\right| \mathcal{V}_{\rho}\left(\left(\Phi \star a_{j}\right)_{b}\right)(x) \chi_{4 B_{j}}(x) \\
& +\mathcal{V}_{\rho}\left(\Phi \star\left(\sum_{j} \lambda_{j} a_{j}\left(b-\langle b\rangle_{B_{j}}\right)\right)\right)(x) \chi_{\left(4 B_{j}\right)}(x) \\
& +\sum_{j}\left|\lambda_{j}\right|\left|b(x)-\langle b\rangle_{B_{j}}\right| \mathcal{V}_{\rho}\left(\Phi \star a_{j}\right)(x) \chi_{\left(4 B_{j}\right)}(x) \\
= & : \sum_{i=1}^{3} I_{i, j}(x) .
\end{aligned}
$$

Firstly, we consider $I_{1, j}(x)$. By Hölder's inequality and Theorem 1.1, we obtain

$$
\begin{aligned}
& \int_{\mathbb{R}^{n}} \mathcal{V}_{\rho}\left(\left(\Phi \star a_{j}\right)_{b}\right)(x) \chi_{4 B_{j}}(x) d x \\
& \lesssim\left(\int_{4 B_{j}} \mathcal{V}_{\rho}\left(\left(\Phi \star a_{j}\right)_{b}\right)(x)^{2} d x\right)^{1 / 2}\left|B_{j}\right|^{1 / 2} \\
& \lesssim\|b\|_{\mathrm{BMO}\left(\mathbb{R}^{\mathrm{n}}\right)}\left|B_{j}\right|^{1 / 2}\left(\int_{B_{j}}\left|a_{j}(x)\right|^{2} d x\right)^{1 / 2} \\
& \lesssim\|b\|_{\mathrm{BMO}\left(\mathbb{R}^{\mathrm{n}}\right)}\left|B_{j}\right|^{1 / 2}\left|B_{j}\right|^{-1 / 2}=\|b\|_{\mathrm{BMO}\left(\mathbb{R}^{\mathrm{n}}\right)}
\end{aligned}
$$

Thus, by the Chebyshev inequality and $\sum_{j}\left|\lambda_{j}\right| \leq 2\|f\|_{H^{1}\left(\mathbb{R}^{n}\right)}$,

$$
\left|\left\{x \in \mathbb{R}^{n}: I_{1, j}(x)>\alpha / 3\right\}\right|
$$




$$
\begin{aligned}
& \leq \frac{3}{\alpha} \sum_{j}\left|\lambda_{j}\right| \int_{\mathbb{R}^{n}} \mathcal{V}_{\rho}\left(\left(\Phi \star a_{j}\right)_{b}\right)(x) \chi_{4 B_{j}}(x) d x \\
& \lesssim \frac{1}{\alpha}\|b\|_{\mathrm{BMO}\left(\mathbb{R}^{\mathrm{n}}\right)}\|f\|_{H^{1}\left(\mathbb{R}^{n}\right)} .
\end{aligned}
$$

Next, we pay our attention to $I_{2, j}(x)$. By Lemma 2.7 and

$$
\left(\frac{1}{\left|B_{j}\right|} \int_{B_{j}}\left|b(x)-\langle b\rangle_{B_{j}}\right|^{2} d x\right)^{1 / 2} \lesssim\|b\|_{\mathrm{BMO}\left(\mathbb{R}^{\mathrm{n}}\right)}
$$

we deduce that

$$
\begin{aligned}
\mid\{x & \left.\in \mathbb{R}^{n}: I_{2, j}(x)>\alpha / 3\right\} \mid \\
& \lesssim \frac{1}{\alpha}\left\|\sum_{j} \lambda_{j}\left(b-\langle b\rangle_{B_{j}}\right) a_{j}\right\|_{L^{1}\left(\mathbb{R}^{n)}\right.} \\
& \leq \frac{1}{\alpha} \sum_{j}\left|\lambda_{j}\right|\left\|\left(b-\langle b\rangle_{B_{j}}\right) a_{j}\right\|_{L^{1}\left(\mathbb{R}^{n}\right)} \\
& \leq \frac{1}{\alpha} \sum_{j}\left|\lambda_{j}\right|\left(\int_{B_{j}}\left|b(x)-\langle b\rangle_{B_{j}}\right|^{2} d x\right)^{1 / 2}\left(\int_{B_{j}}\left|a_{j}(x)\right|^{2} d x\right)^{1 / 2} \\
& \lesssim \frac{1}{\alpha} \sum_{j}\left|\lambda_{j}\right|\|b\|_{\mathrm{BMO}\left(\mathbb{R}^{\mathrm{n}}\right)}\left|B_{j}\right|^{1 / 2}\left|B_{j}\right|^{-1 / 2} \\
& \lesssim \frac{1}{\alpha}\|b\|_{\mathrm{BMO}\left(\mathbb{R}^{\mathrm{n}}\right)}\|f\|_{H^{1}\left(\mathbb{R}^{n}\right)} .
\end{aligned}
$$

Finally, we deal with $I_{3, j}(x)$. We make use of (3.1) and the vanishing property of $a_{j}$ and Minkowski's inequality to obtain

$$
\begin{aligned}
& \mathcal{V}_{\rho}\left(\Phi \star a_{j}\right)(x) \\
& =\sup _{\left\{t_{k}\right\} \downarrow}\left(\sum_{k} \mid \int_{\mathbb{R}^{n}}\left[\left(\phi_{t_{k}}(x-y)-\phi_{t_{k+1}}(x-y)\right)\right.\right. \\
& \left.\left.\quad-\left(\phi_{t_{k}}\left(x-x_{j}\right)-\phi_{t_{k+1}}\left(x-x_{j}\right)\right)\right]\left.a_{j}(y) d y\right|^{\rho}\right)^{1 / \rho} \\
& \leq \int_{B_{j}}\left|a_{j}(y)\right|\left\|\left\{\phi_{t}(x-y)-\phi_{t}\left(x-x_{j}\right)\right\}_{t>0}\right\|_{\mathcal{V}_{\rho}} d y \\
& \lesssim \int_{B_{j}}\left|a_{j}(y)\right| \frac{\left|y-x_{j}\right|}{\left|x-x_{j}\right|^{n+1}} d y .
\end{aligned}
$$

Observe that

$$
\int_{B_{j}}\left|a_{j}(y)\right| d y \leq 1
$$

From (3.5), we deduce that

$$
\int_{\mathbb{R}^{n}}\left|b(x)-\langle b\rangle_{B_{j}}\right| \mathcal{V}_{\rho}\left(\Phi \star a_{j}\right)(x) \chi_{\left(4 B_{j}\right)}(x) d x
$$




$$
\begin{aligned}
& \leq \sum_{l=2}^{\infty} \int_{2^{l+1} B_{j} \backslash 2^{l} B_{j}}\left|b(x)-\langle b\rangle_{B_{j}}\right| \int_{B_{j}} \frac{\left|y-x_{j}\right|}{\left|x-x_{j}\right|^{n+1}}\left|a_{j}(y)\right| d y d x \\
& \lesssim \sum_{l=2}^{\infty} \int_{2^{l+1} B_{j}} \frac{r_{j}}{\left(2^{l+1} r_{j}\right)^{n+1}}\left|b(x)-\langle b\rangle_{B_{j}}\right| d x \\
& \lesssim \sum_{l=2}^{\infty} 2^{-l}\left(\frac{1}{\left|2^{l+1} B_{j}\right|} \int_{2^{l+1} B_{j}}\left|b(x)-\langle b\rangle_{2^{l+1} B_{j}}\right| d x+\left|\langle b\rangle_{2^{l+1} B_{j}}-\langle b\rangle_{B_{j}}\right|\right) \\
& \leq \sum_{l=2}^{\infty} 2^{-l}\left[1+2^{n}(l+1)\right]\|b\|_{\mathrm{BMO}\left(\mathbb{R}^{\mathrm{n}}\right)} \sim\|b\|_{\mathrm{BMO}\left(\mathbb{R}^{\mathrm{n}}\right) .}
\end{aligned}
$$

Therefore,

$$
\begin{aligned}
\mid\{x & \left.\in \mathbb{R}^{n}: I_{3, j}(x)>\alpha / 3\right\} \mid \\
& \leq \frac{3}{\alpha} \int_{\mathbb{R}^{n}} \sum_{j}\left|\lambda_{j}\right|\left|b(x)-\langle b\rangle_{B_{j}}\right| \mathcal{V}_{\rho}\left(\Phi \star a_{j}\right)(x) \chi_{\left(4 B_{j}\right)} c(x) d x \\
& \lesssim \frac{1}{\alpha} \sum_{j}\left|\lambda_{j}\right|\|b\|_{\mathrm{BMO}\left(\mathbb{R}^{\mathrm{n}}\right)} \\
& \lesssim \frac{1}{\alpha}\|b\|_{\mathrm{BMO}\left(\mathbb{R}^{\mathrm{n}}\right)}\|f\|_{H^{1}\left(\mathbb{R}^{n}\right)} .
\end{aligned}
$$

Combining with (3.2), (3.3), (3.4), and (3.6), we get the desired result.

Finally, we prove Theorem 1.3.

Proof of Theorem 1.3 From Lemma 2.5 and Lemma 2.7, we only need to check condition (2.1). For any $\psi$-atom $a$ of $H^{1}\left(\mathbb{R}^{n}\right)$, suppose that supp $a \subset Q$ and $x_{0}$ is the center of cube $Q$. By Hölder's inequality, $\|a\|_{L^{2}(Q)} \leq|Q|^{-1 / 2}$, and the $L^{p}$-boundedness of $\mathcal{V}_{\rho}(\Phi \star a)$, we have

$$
\begin{aligned}
\int_{4 Q}\left|b(x)-\langle b\rangle_{Q}\right| \mathcal{V}_{\rho}(\Phi \star a)(x) d x & \lesssim\left(\int_{4 Q}\left|b(x)-\langle b\rangle_{Q}\right|^{2} d x\right)^{1 / 2}\|a\|_{L^{2}\left(\mathbb{R}^{n}\right)} \\
& \lesssim\|b\|_{\mathrm{BMO}\left(\mathbb{R}^{\mathrm{n}}\right)}
\end{aligned}
$$

On the other hand, using (3.5), $\|a\|_{L^{2}(Q)} \leq|Q|^{-1 / 2}$, and Hölder's inequality, we have

$$
\begin{aligned}
& \int_{2^{j+1} Q \backslash 2 j Q}\left|b(x)-\langle b\rangle_{Q}\right| \mathcal{V}_{\rho}(\Phi \star a)(x) d x \\
& \quad \lesssim \int_{2^{j+1} Q \backslash 2^{j} Q}\left|b(x)-\langle b\rangle_{Q}\right| \int_{Q} \frac{\left|y-x_{0}\right|}{\left|x-x_{0}\right|^{n+1}}|a(y)| d y d x \\
& \quad \lesssim|Q|^{1 / n} \int_{2^{j+1} Q \backslash 2^{j} Q}\left|b(x)-\langle b\rangle_{Q}\right|\left|x-x_{0}\right|^{-n-1} \int_{Q}|a(y)| d y d x \\
& \quad \leq|Q|^{1 / n} \int_{2^{j+1} Q \backslash 2^{j} Q}\left|b(x)-\langle b\rangle_{Q}\right|\left|x-x_{0}\right|^{-n-1} d x .
\end{aligned}
$$


Now, in fact, we get that

$$
\begin{aligned}
& \int_{(4 Q)^{c}}\left|b(x)-\langle b\rangle_{Q}\right| \mathcal{V}_{\rho}(\Phi \star a)(x) d x \\
& \lesssim \sum_{j=2}^{\infty}|Q|^{1 / n} \int_{2^{j+1} Q \backslash 2^{j} Q}\left|b(x)-\langle b\rangle_{Q}\right|\left|x-x_{0}\right|^{-n-1} d x \\
& \lesssim \sum_{j=2}^{\infty} \frac{|Q|^{1 / n}}{\left|2^{j+1} Q\right|^{1 / n}}\left(\frac{1}{\left|2^{j+1} Q\right|} \int_{2^{j+1} Q}\left|b(x)-\langle b\rangle_{2^{j+1} Q}\right| d x+\left|\langle b\rangle_{2^{j+1} Q}-\langle b\rangle_{Q}\right|\right) \\
& \lesssim\|b\|_{\mathrm{BMO}\left(\mathbb{R}^{\mathrm{n}}\right) .}
\end{aligned}
$$

Then, by (3.7) and (3.8), we get (2.1). This completes our proof.

\section{Acknowledgements}

The authors thank the referees cordially for their valuable suggestions and comments.

\section{Funding}

This work is supported by the NSF of Shandong Province (GrantNos. ZR2019YQ04, 2020KJI002), President's fund of Minnan Normal University (No. KJ2020020), the scientific research project of The Education Department of Fujian Province (No. JAT200331), and Fujian Key Laboratory of Granular Computing and Applications (Minnan Normal University), China.

\section{Availability of data and materials}

Not applicable.

\section{Competing interests}

The authors declare that they have no competing interests.

\section{Authors' contributions}

All authors contributed equally and significantly in writing this paper. All authors read and approved the final manuscript.

\section{Author details}

'School of Mathematics and Statistics, Minnan Normal University, Zhangzhou 363000, China. ${ }^{2}$ School of Mathematics and Statistics, LinYi University, LinYi 276005, China.

\section{Publisher's Note}

Springer Nature remains neutral with regard to jurisdictional claims in published maps and institutional affiliations.

Received: 17 September 2020 Accepted: 12 July 2021 Published online: 17 August 2021

\section{References}

1. Betancor, J.J., Farina, J.C., Harbour, E., Rodriguez-Mesa, L.: $L^{p}$-boundedness properties of variation operators in the Schrödinger setting. Rev. Mat. Complut. 26(2), 485-534 (2013)

2. Bourgain, J.: Pointwise ergodic theorems for arithmetric sets. Publ. Math. Inst. Hautes Études Sci. 69(1), 5-45 (1989)

3. Bourgain, J., Mirek, M., Stein, E.M., Wróbel, B.A.: On dimension-free variational inequalities for averaging operators in $\mathbb{R}^{d}$. Geom. Funct. Anal. 28(1), 58-99 (2018)

4. Bui, T.A.: Boundedness of variation operators and oscillation operators for certain semigroups. Nonlinear Anal. 106, 124-137 (2014)

5. Campbell, J.T., Jones, R.L., Reinhold, K., Wierdl, M.: Oscillations and variation for the Hilbert transform. Duke Math. J. 105(1), 59-83 (2000)

6. Campbell, J.T., Jones, R.L., Reinhold, K., Wierdl, M.: Oscillations and variation for singular integrals in higher dimensions. Trans. Am. Math. Soc. 355(5), 2115-2137 (2003)

7. Chen, Y., Ding, Y., Hong, G., Liu, H.: Variational inequalities for the commutators of rough operators with BMO functions. J. Funct. Anal. 275(8), 2446-2475 (2018)

8. Coifman, R.R., Rochberg, R., Weiss, G.: Factorization theorems for Hardy spaces in several variables. Ann. Math. (2) 103(3), 611-635 (1976)

9. Crescimbeni, R., Macías, R.A., Menárguez, T., Torrea, J.L., Viviani, B.: The $\rho$-variation as an operator between maximal operators and singular integrals. J. Evol. Equ. 9(1), 81-102 (2009)

10. Ding, Y., Hong, G., Liu, H.: Jump and variational inequalities for rough operators. J. Fourier Anal. Appl. 23(3), 679-711 (2017)

11. Duong, X.T., Li, J., Yang, D.: Variation of Calderón-Zygmund operators with matrix weight. arXiv:1811.11324v2

12. Fefferman, C., Stein, E.M.: $H^{P}$ spaces of severable variables. Acta Math. 129, 137-193 (1972)

13. Gillespie, T.A., Torrea, J.L.: Dimension free estimates for the oscillation of Riesz transform. Isr. J. Math. 141, 125-144 (2004) 
14. Jones, R.L., Seeger, A., Wright, J.: Strong variational and jump inequalities in harmonic analysis. Trans. Am. Math. Soc 360(12), 6711-6742 (2008)

15. Ky, L.D.: Bilinear decompositions and commutators of singular integral operators. Trans. Am. Math. Soc. 365(6), 2931-2958 (2013)

16. Lépingle, D.: La variation d'order p des semi-martingales. Z. Wahrscheinlichkeitstheor. Verw. Geb. 36(4), 295-316 (1976)

17. Liu, F., Jhang, S., Oh, S.-K., Fu, Z.: Variation inequalities for one-sided singular integrals and related commutators. Mathematics 7(10), 876 (2019)

18. Liu, F., Wu, H.: A criterion on oscillation and variation for the commutators of singular integrals. Forum Math. 27, 77-97 (2015)

19. Liu, H.: Variational characterization of $H^{P}$. Proc. R. Soc. Edinb., Sect. A 149(5), 1123-1134 (2019)

20. Ma, T., Torrea, J.L., Xu, Q.: Weighted variation inequalities for differential operators and singular integrals. J. Funct. Anal. 268(2), 376-416 (2015)

21. Mirek, M., Stein, E.M., Trojan, B.: $\mathbb{P}\left(\mathbb{Z}^{d}\right)$-estimates for discrete operators of Radon type: variational estimates. Invent. Math. 209(3), 665-748 (2017)

22. Paluszyński, M.: Characterization of Lipschitz spaces via commutators of Coifman, Rocherberg and Weiss; a multiplier theorem for the semigroup of contractions. Thesis, Washington University (1992)

23. Pérez, C.: Endpoint estimates for commutators of singular integral operators. J. Funct. Anal. 128(1), 163-185 (1995)

24. Stein, E.M.: Harmonic Analysis: Real-Variable Methods, Orthogonality, and Oscillatory Integrals. Princeton University Press, Princeton (1993)

25. Tang, L., Zhang, Q.: Variation operators for semigroups and Riesz transforms acting on weighted $L^{p}$ and BMO spaces in the Schrödinger setting. Rev. Mat. Complut. 29(3), 559-621 (2016)

26. Wu, H., Yang, D., Zhang, J.: Oscillation and variation for semigroups associated with Bessel operators. J. Math. Anal. Appl. 443(2), 848-867 (2016)

27. Wu, H., Yang, D., Zhang, J.: Oscillation and variation for the Riesz transform associated with Bessel operators. Proc. R. Soc. Edinb., Sect. A 149(1), 169-190 (2019)

28. Zhang, J., Wu, H.: Oscillation and variation inequalities for the commutators of singular integrals with Lipschitz functions. J. Inequal. Appl. 2015, 214 (2015)

29. Zhang, J., Wu, H.: Oscillation and variation inequalities for singular integrals and commutators on weighted Morrey spaces. Front. Math. China 11(2), 423-447 (2016)

\section{Submit your manuscript to a SpringerOpen ${ }^{\circ}$ journal and benefit from:}

- Convenient online submission

- Rigorous peer review

- Open access: articles freely available online

- High visibility within the field

- Retaining the copyright to your article

Submit your next manuscript at $>$ springeropen.com 